\title{
Management of failed Chiari decompression and intrasyringeal hemorrhage in Noonan syndrome: illustrative cases
}

\author{
Cody J. Falls, BS, ${ }^{1}$ Paul S. Page, MD, ${ }^{2}$ Garret P. Greeneway, $M D^{2},{ }^{2}$ Daniel K. Resnick, MD, ${ }^{2}$ and James A. Stadler III, $\mathrm{MD}^{2}$ \\ ${ }^{1}$ University of Wisconsin School of Medicine and Public Health, Madison, Wisconsin; and ${ }^{2}$ Department of Neurological Surgery, University of Wisconsin School of Medicine and \\ Public Health, Madison, Wisconsin
}

\begin{abstract}
BACKGROUND Noonan syndrome (NS) is a rare genetic RASopathy with multisystem implications. The disorder is typically characterized by short stature, distinctive facial features, intellectual disability, developmental delay, chest deformity, and congenital heart disease. NS may be inherited or arise secondary to spontaneous mutations of genes in the Ras/mitogen activated protein kinase signaling pathways.

OBSERVATIONS Numerous case reports exist detailing the association between NS and Chiari I malformation (CM-I), although this relationship has not been fully established. Patients with NS who present with CM-I requiring operation have shown high rates reoperation for failed decompression. The authors reported two patients with NS, CM-I, and syringomyelia who had prior posterior fossa decompressions without syrinx improvement. Both patients received reoperation with successful outcomes.
\end{abstract}

LESSONS The authors highlighted the association between NS and CM-I and raised awareness that patients with these disorders may be at higher risk for failed posterior fossa decompression, necessitating reoperation.

https://thejns.org/doi/abs/10.3171/CASE21625

KEYWORDS Chiari decompression; Chiari malformation; Noonan syndrome; RASopathy; reoperation; syringomyelia

Noonan syndrome (NS) is a rare genetic disorder affecting between 1 in 1,000 and 1 in 2,500 individuals worldwide. ${ }^{1} \mathrm{NS}$ may affect more males than females, and it displays a wide array of features depending on the causative gene mutation. ${ }^{2}$ This disorder is most commonly inherited in an autosomal dominant fashion, although autosomal recessive inheritance and spontaneous mutations are also implicated for certain subpopulations. PTPN11 is the most frequent gene mutation in NS and is found in approximately $50 \%$ of affected individuals. ${ }^{1}$

NS shares phenotypic similarities with other disorders involving Ras/mitogen activated protein kinase gene mutations (RASopathies), including cardiofaciocutaneous, neurofibromatosis type 1, Costello, and Legius syndromes. ${ }^{2}$ NS is most commonly characterized by short stature, distinctive facial features, intellectual disability, developmental delay, chest deformity, and congenital heart disease, although it can affect nearly all organ systems. ${ }^{2}$ Multiple case reports have described patients with NS, Chiari 1 malformations
(CM-I), and syringomyelia, although the small population size prevents full characterization of this association. ${ }^{3-8}$

\section{Illustrative Cases \\ Case 1}

A 25-year-old man with NS was found to have CM-I and associated syringomyelia after initial presentation with left leg sensory loss. He underwent posterior fossa decompression with duraplasty and had postoperative improvement in his symptoms. One year after surgery, he reported temperature sensory loss in the left arm, which prompted repeat magnetic resonance imaging (MRI), which demonstrated adequate posterior fossa decompression and C1 laminectomy but persistence of his syrinx with an associated cyst that did not appear fenestrated (Fig. 1). Despite surgical intervention being offered, the family elected for conservative therapy, and his symptoms gradually improved.

ABBREVIATIONS CM-I = Chiari I malformation; CSF = cerebrospinal fluid; MRI = magnetic resonance imaging; $N S=$ Noonan syndrome INCLUDE WHEN CITING Published January 24, 2022; DOI: 10.3171/CASE21625.

SUBMITTED November 1, 2021. ACCEPTED November 19, 2021.

(C) 2022 The authors, CC BY-NC-ND 4.0 (http://creativecommons.org/licenses/by-nc-nd/4.0/). 

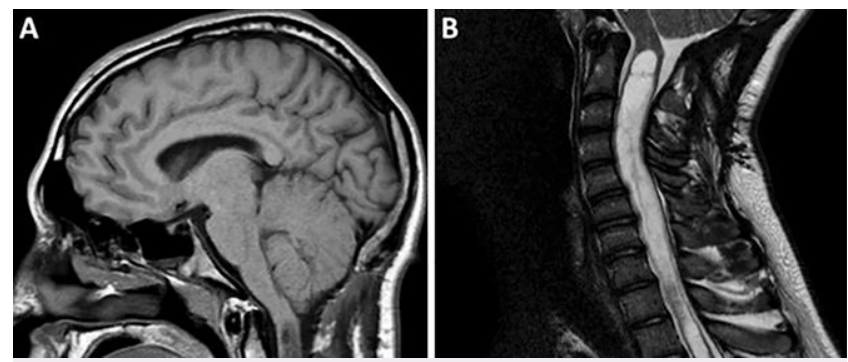

FIG. 1. Case 1. A: T1 sagittal brain MRI at initial 1-year postoperative visit demonstrating adequate posterior fossa decompression. B: T2 sagittal cervical spine MRI at initial 1-year postoperative visit demonstrating persistent syrinx.

Five years later, he presented to an outside hospital with acute bilateral shoulder pain, low back pain, diffuse loss of sensation, acute urinary retention, clawing of the hands, and gait ataxia. Repeat MRI revealed an intrasyringeal hemorrhage (Fig. 2). Notably, his syrinx had decreased in size, extending from the cervicomedullary junction to the T2 spinal level. There was also evidence of syrinx dilatation at the rostral end when compared to his postoperative imaging 5 years earlier. The patient received an extensive hematology evaluation that was ultimately unrevealing. His symptoms improved significantly with steroid treatment, and he was subsequently discharged home. At follow-up with us in clinic 1 week later, he demonstrated a stable neurological examination. At that point, the decision was made to pursue repeat MRI with cine sequences once the blood had cleared to maximize visualization and better understand his cerebrospinal fluid (CSF) flow dynamics for preoperative planning.

Six weeks later, cine MRI demonstrated CSF flow obstruction adjacent to the syrinx in the upper cervical spine and expected resolution of blood products. Additionally, the syrinx did demonstrate a caudal cyst that appeared to be noncommunicating with the remainder of the syrinx. The patient still reported pain after a period of
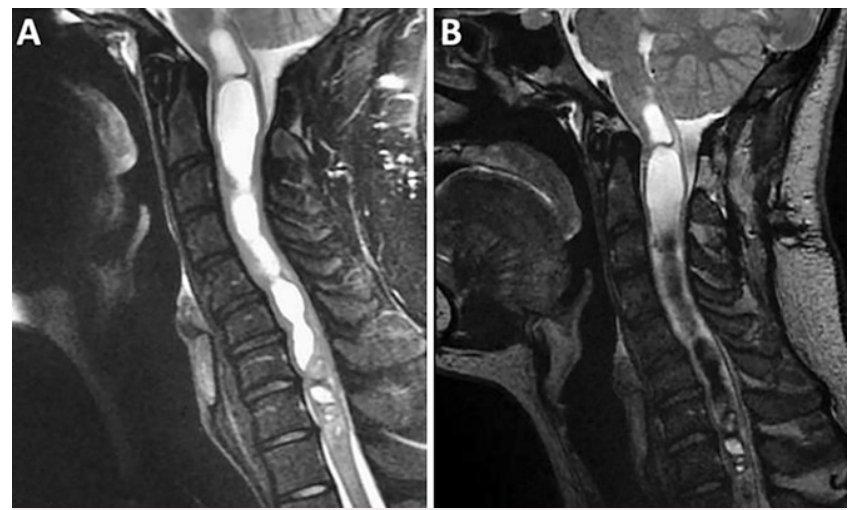

FIG. 2. Case 1. A: T2 sagittal cervical spine MRI at 6-year postoperative visit demonstrating overall decreased syrinx size, now extending from the cervicomedullary junction to the T2 spinal level. There is evidence of rostral syrinx dilation relative to the overall cervical syrinx size in comparison to postoperative imaging 5 years earlier. B: T2 sagittal cervical spine MRI with nongated CUBE sequencing at 6-year postoperative visit demonstrating dark $\mathrm{T} 2$ signal within the syrinx, consistent with intrasyringeal hemorrhage.
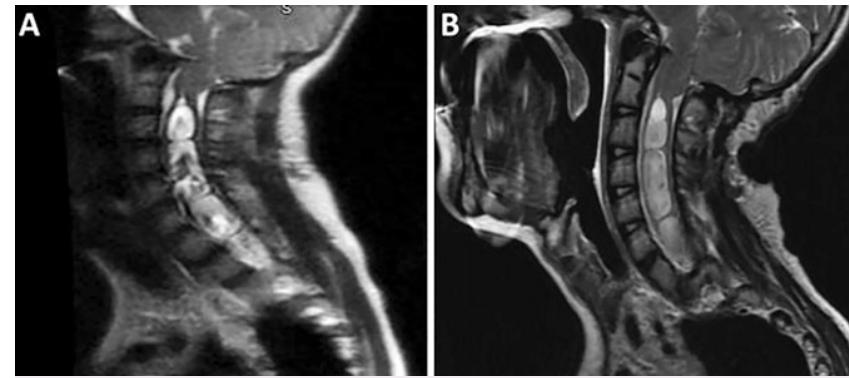

FIG. 3. Case 2. A: T2 sagittal spine MRI obtained postoperatively after initial posterior fossa decompression demonstrating syringomyelia. B: T2 sagittal spine MRI obtained approximately 5 years postoperatively after initial posterior fossa decompression demonstrating unchanged syringomyelia.

conservative management and therefore received repeat posterior fossa decompression with intradural exploration and expansile duraplasty. There was intraoperative evidence of scar tissue within the foramen of Magendie that was fenestrated. A caudal cyst was also appreciated within the upper cervical syrinx, which seemingly did not communicate with the subarachnoid space. The caudal cyst was fenestrated and drained, resulting in a rush of hemosiderinstained fluid. There were no intraoperative or postoperative complications, and the patient was discharged home on postoperative day 2. Two months after surgery, he continued to demonstrate good relief of his symptoms. His neck and back pain resolved, he no longer experienced urinary retention, his hands were no longer clawed, and he had no issues with gait.

However, 1 month later, his neck and back pain returned, and he began having emesis and temporo-occipital headaches. MRI at that time revealed expansion of the upper portion of the syrinx into the brainstem. In addition, the ventricles were enlarged with periventricular edema. After discussion with family, an endoscopic third ventriculostomy was performed. Prior to that occurrence there had been no clinical concern for elevated intracranial pressure or need for CSF diversion. One month postoperatively, the patient has complete resolution of his neck pain and headaches and continues to demonstrate no other neurological symptoms.
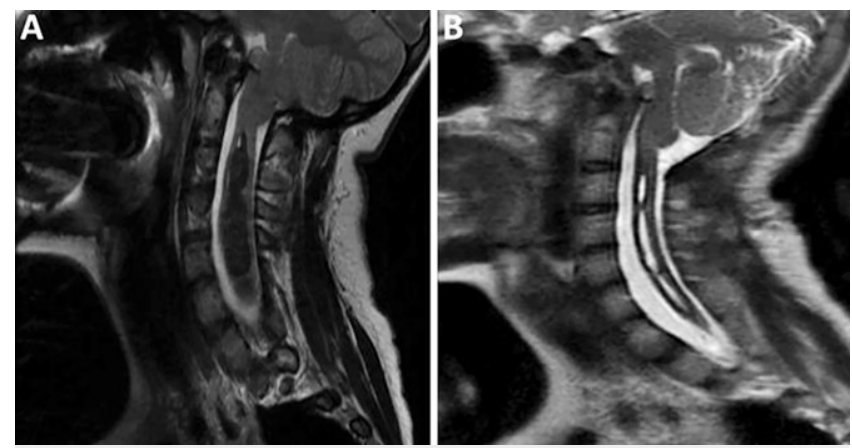

FIG. 4. Case 2. A: T2 sagittal spine MRI obtained approximately 6 years postoperatively after initial posterior fossa decompression demonstrating persistent syringomyelia with evidence of dark T2 signal within the syrinx, concerning for intrasyringeal hemorrhage. B: T2 sagittal spine MRI obtained 5 months postoperatively after second posterior fossa decompression demonstrating decreased overall syrinx size. 


\section{Case 2}

A 16-year-old girl with a known history of NS, tethered cord, scoliosis, CM-I, and holocord syringomyelia presented with rapid progression of her scoliosis. She had a history of a tethered cord release 7 years earlier. Lack of syrinx improvement prompted posterior fossa decompression with $\mathrm{C} 1$ laminectomy and expansile duraplasty 8 months later. Postoperatively, the syrinx remained stable, and she had no clinical progression of symptoms.

Approximately 5 years after her posterior fossa decompression, her clinical examination remained stable, but she was noted to have rapid scoliosis progression, which was confirmed radiographically. MRI showed unchanged holocord syringomyelia, and CSF flow studies were notable for ventralization and relative decrease of CSF flow at the level of the foramen magnum (Fig. 3). Surgery was recommended but deferred per patient and family preferences. Repeat MRI obtained 1 year later demonstrated no improvement of her syrinx and continued progression of her scoliosis. Notably, T2 MRI sequences were remarkable for intrasyringeal hemorrhage (Fig. 3); she was clinically asymptomatic from this. The patient subsequently received an extensive hematology evaluation that revealed no specific coagulation disorder but was concerning for multiple low coagulation factors, specifically VIII, IX, IX, and fibrinogen.

Three weeks later, the patient had reoperation in which she received extension of her original posterior fossa decompression via

TABLE 1. Literature review describing nine cases of Noonan syndrome and Chiari malformation with pertinent operative history

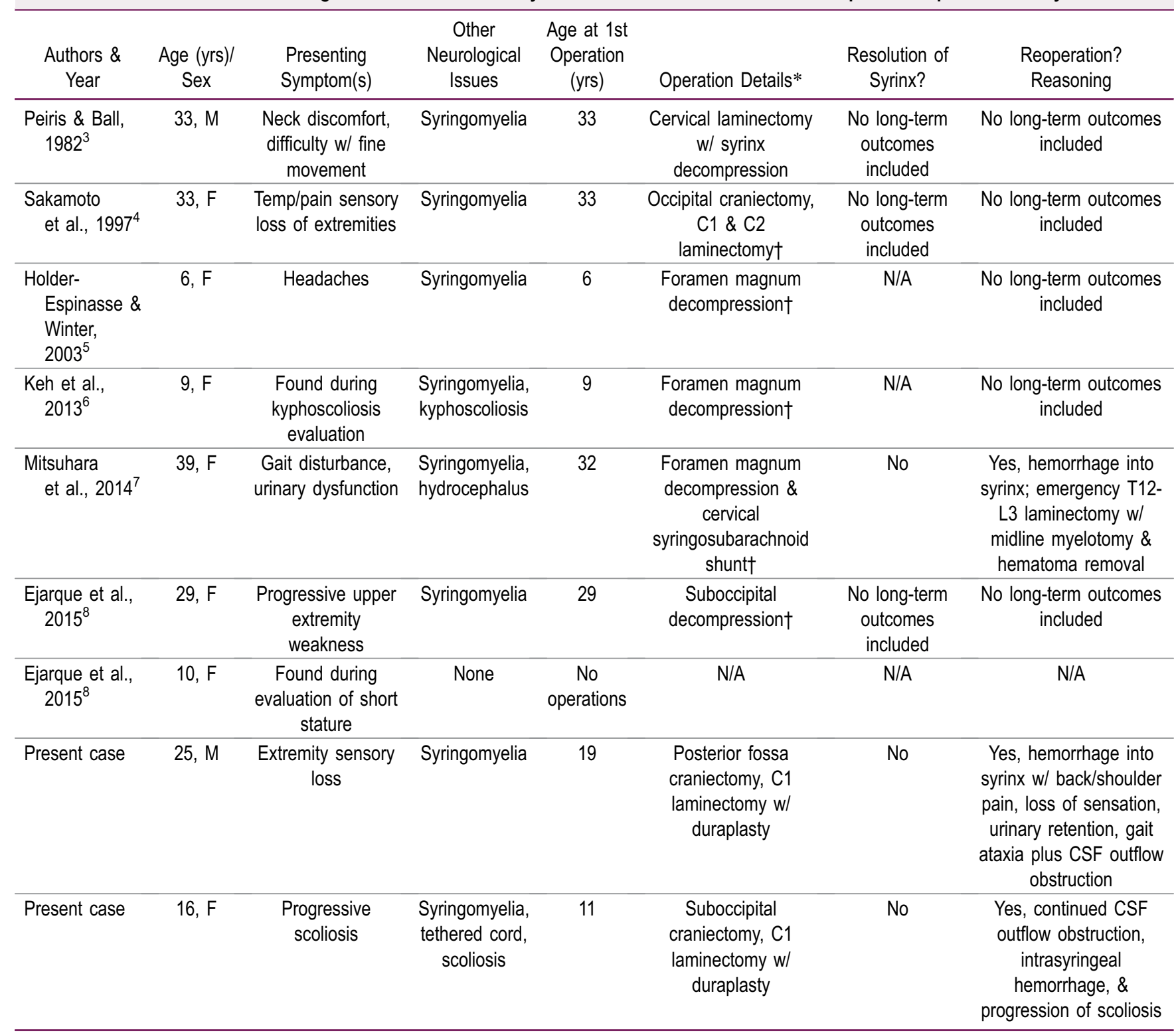

$\mathrm{N} / \mathrm{A}=$ not applicable.

* Exactly as provided in article.

$\uparrow$ No mention of intradural exploration and/or duroplasty. 
C2 laminectomy to ensure adequate caudal decompression with complete lysis of adhesions as well as repeat expansile duraplasty. She received cryoprecipitate and fresh frozen plasma perioperatively. There were no intraoperative or postoperative complications, and the patient was discharged on postoperative day 5 . MRI revealed a decrease in the size of her syrinx 5 months after surgery (Fig. 4). She received staged correction of the spinal deformity approximately 1 year after posterior fossa decompression, and she continues to do well clinically.

\section{Discussion}

\section{Observations}

The association of $\mathrm{CM}-\mathrm{I}$ and RASopathies has previous limited descriptions in the scientific literature. ${ }^{9}$ Despite this, the relationship between NS and CM-I is often underappreciated. There are currently no screening guidelines for asymptomatic patients, and patients with cognitive impairment may have mild symptoms that are unrecognized. ${ }^{6}$ Given the known association, however, CM-I and syringomyelia should be considered as potential factors in any patient with NS and spinal concerns, including scoliosis.

Of the nine reports of patients with NS and CM-I, eight detail patients who required a surgical decompression as a result of their pathology (Table 1). Of these cases, only one report describes long-term outcomes, with that report illustrating a patient requiring reoperation. Two patients with $\mathrm{NS}, \mathrm{CM}-\mathrm{I}$, and syringomyelia were recently evaluated at our institution, both of whom demonstrated previous failed posterior fossa decompression and spontaneous intrasyringeal hemorrhage.

At present, these cases highlight the importance of ensuring adequate posterior fossa decompression for the relief of CSF pathway obstruction. Most patients with NS and CM-I present with symptoms secondary to syringomyelia (Table 1), and many of the syrinxes in these patients do not resolve, prompting reoperation. In a meta-analysis by Osborne-Grinter et al. looking at posterior fossa reoperation rates in patients with $\mathrm{CM}-\mathrm{I}$ in a broad population, $0.3 \%-8 \%$ of patients required reoperation, largely depending on whether the arachnoid was dissected or preserved. ${ }^{10}$ With our present cases being added to the literature, all three patients with observed long-term outcomes and three of nine total reported cases required reoperation. Although a larger sample size is needed for formal conclusions, this increased rate of reoperation seen in patients with NS and CM-I raises the concern that these patients are at high risk for failure of surgical decompression over time.

Additionally, these cases also highlight the possibility of spontaneous intrasyringeal hemorrhage, an otherwise rare phenomenon that has now been seen in three patients with NS. The etiology of these hemorrhages is unclear; patients with NS may have unidentified bleeding disorders, although none were identified in our patients on hematological examination. ${ }^{11}$ Given the atypical scar formation and apparent increased risk for reoperation, primary intradural or intrasyringeal hemorrhage may result in elevated syrinx pressure, leading to rupture of the vasculature in the syrinx wall. Although intrasyringeal hemorrhage, or Gowers syndrome, has been reported in the literature, it is a rare occurrence that has been reported fewer than 20 times in the known medical literature. ${ }^{9}$ Regardless, providers should be aware of the potential need for repeat decompression and the risk for intrasyringeal hemorrhage in patients with NS.

\section{Lessons}

A continuously growing body of evidence supports the relationship between NS and CM-I. Of the reported cases, most of these patients end up presenting clinically with symptoms related to syringomyelia, ultimately prompting surgical decompression. Reoperation rates in these patients appear to be higher than the standard population. Patients with $\mathrm{NS}, \mathrm{CM}-\mathrm{I}$, and syringomyelia may also have risk for intrasyringeal hemorrhage. The reasons for these findings and potential methods to decrease reoperation rates in NS patients remain to be determined.

\section{References}

1. Allanson JE. Noonan syndrome. In: The NORD Guide to Rare Disorders. Lippincott, Williams and Wilkins; 2003:722-723.

2. Rauen KA. The RASopathies. Annu Rev Genomics Hum Genet. 2013;14:355-369.

3. Peiris A, Ball MJ. Chiari (type 1) malformation and syringomyelia in a patient with Noonan's syndrome. J Neurol Neurosurg Psychiatry. 1982;45(8):753-754.

4. Sakamoto T, Suzuki R, Hazama Y, Sekino $H$. Chiari malformation type 1 and syringomyelia in a patient with Noonan syndrome: a case report. Jpn J Neurosurg. 1997;6(8):565-569.

5. Holder-Espinasse M, Winter RM. Type 1 Arnold-Chiari malformation and Noonan syndrome. A new diagnostic feature? Clin Dysmorphol. 2003;12(4):275.

6. Keh YS, Abernethy L, Pettorini B. Association between Noonan syndrome and Chiari I malformation: a case-based update. Childs Nerv Syst. 2013;29(5):749-752.

7. Mitsuhara T, Yamaguchi S, Takeda M, Kurisu K. Gowers' intrasyringeal hemorrhage associated with Chiari type I malformation in Noonan syndrome. Surg Neurol Int. 2014;5:6.

8. Ejarque I, Millán-Salvador JM, Oltra S, Pesudo-Martínez JV Beneyto M, Pérez-Aytés A. Arnold-Chiari malformation in Noonan syndrome and other syndromes of the RAS/MAPK pathway. Article in Spanish. Rev Neurol. 2015;60(9):408-412.

9. Saletti V, Viganò I, Melloni G, Pantaleoni C, Vetrano IG, Valentini LG. Chiari I malformation in defined genetic syndromes in children: are there common pathways? Childs Nerv Syst. 2019;35(10): 1727-1739.

10. Osborne-Grinter M, Arora M, Kaliaperumal C, Gallo P. Posterior fossa decompression and duraplasty with and without arachnoid preservation for the treatment of adult Chiari malformation type 1: a systematic review and meta-analysis. World Neurosurg. 2021;151:e579-e598.

11. Nugent DJ, Romano AA, Sabharwal S, Cooper DL. Evaluation of bleeding disorders in patients with Noonan syndrome: a systematic review. J Blood Med. 2018;9:185-192.

\section{Disclosures}

Dr. Resnick reported other from NIDUS outside the submitted work. No other disclosures were reported.

\section{Author Contributions}

Conception and design: Page, Falls, Greeneway, Stadler. Acquisition of data: all authors. Analysis and interpretation of data: Falls, Greeneway. Drafting the article: Page, Falls, Greeneway. Critically revising the article: Page, Falls, Resnick, Stadler. Reviewed submitted version of manuscript: Page, Falls, Resnick, Stadler. Statistical analysis: Page. Administrative/technical/material support: Stadler. Study supervision: Page, Stadler.

\section{Correspondence}

Paul S. Page: University of Wisconsin School of Medicine and Public Health, Madison, WI. paulsamuelpage@gmail.com. 